\title{
Detection of joint space narrowing in hand radiographs
}

\section{J.A. Kauffman ${ }^{1}$, C.H. Slump ${ }^{1}$, H.J. Bernelot Moens ${ }^{2}$}

Signals and Systems Group

Department of Electrical Engineering, Mathematics and Computer Science

University of Twente, Enschede, http://www.sas.el.utwente.nl, email: j.a.kauffman@utwente.nl

Department of Rheumatology

Ziekenhuis Groep Twente, Hengelo/Almelo

\section{University of Twente Enschede - The Netherlands}

\section{Introduction}

\section{Research goal}

Joint damage assessment in hand radiographs to determine progression of rheumatoid arthritis.

\section{Joint space narrowing}

Inflammation of the synovial membrane releases enzymes that break down cartilage. This effect can be monitored indirectly by measuring the distance between the joint margins: the joint space width (JSW).

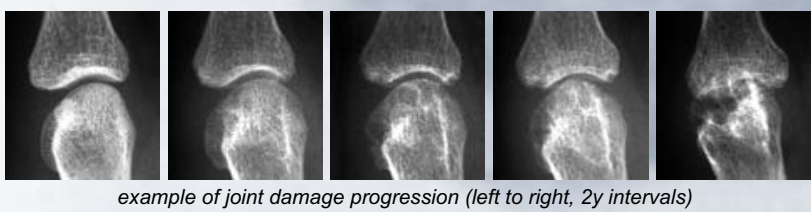

Problems with manual methods

口 intra/inter-reader variability

a time consuming

$\square$ missing golden standard

Proposed solution

Automated detection of joint margins with high reproducibility.

\section{Margin characteristics}

\section{Training set}

50 hand radiographs

- 8 locations: metacarpophalangeal joints $2-5$ and proximal

interphalangeal joints 2-5

a manually outlined joint margins

Active shape model [1]

a 25 equidistant points per margin over $6 \mathrm{~mm}$

- 8 modes of variation

Profile characterization

- 25 intensity profiles perpendicular to margin

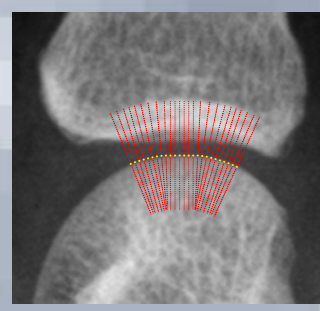

intensity profile lines

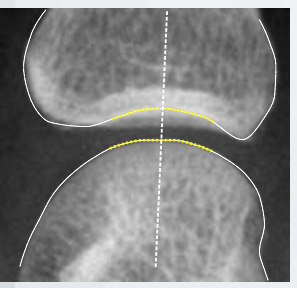

- 15 samples per $\mathrm{mm}$

口 mean and variance estimation

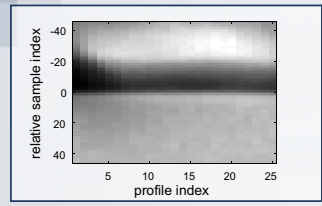

intensity profiles of proximal margin

\section{Search method}

1. Initialization by 8 points on proximal and distal ends of proximal phalanges (PP) Automated initialization with segmentation method [2]

2. Initial estimate of margin is mean of shape model. Orientation is aligned with PP-axis.

3. Profiles are scanned perpendicular to margin.

4. Mahalanobis distance determines likely margin locations in cost matrix.

5. Minimum cost path is calculated with dynamic programming method.

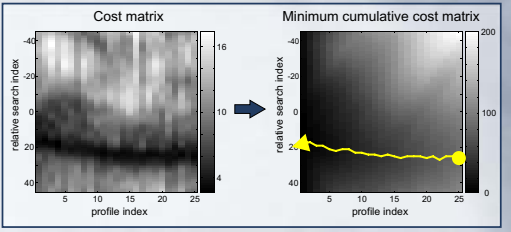

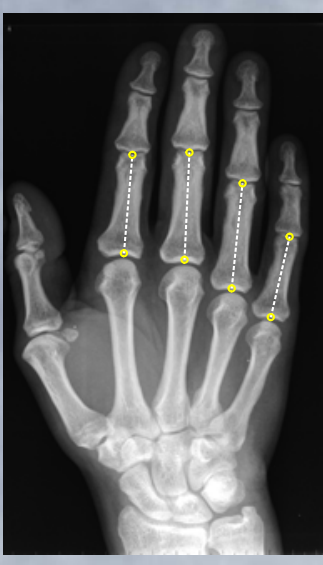

initialization points

6. New margin points are projected into model space and back to find plausible shape.

7. Repeat steps 3-6 4x to reach convergence. calculation of minimum cost path

\section{Conclusions}

- Robust and precise method for visible joint margins. Fails in cases of severe joint damage.

- Automated method has better reproducibility and is more accurate than manual method.

- Shape model constraints prevent detection of false margins, but also limit the detection of unusual margin shapes.

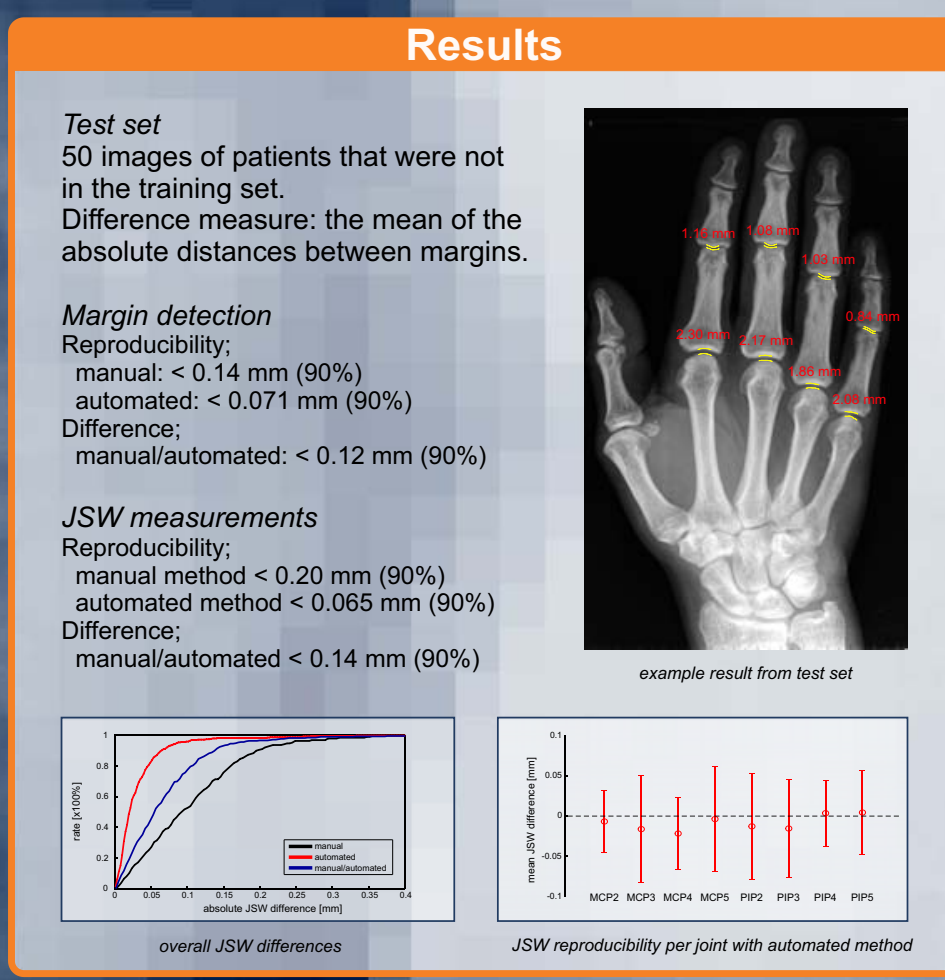

\section{References}

[1] Cootes, T. F., Hill, A., Taylor, C. J., and Haslam, J., "Use of active shape models for locating structure in

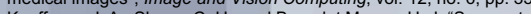

nd radiographs with Image Processing 5747-182.

3] Amini, A. A., Weymouth, T. E., and Jain, R. C., "Using dynamic programming for solving variational problems in vision", Pattern Analysis and Machine Intelligence, IEEE Transactions on, vol. 12, no. 9, pp. 